Cox multivariate analysis identified that presence PD-synovitis at baseline was associated with risk of radiographic progression at 4 years ( $\mathrm{HR} 3,6895 \% \mathrm{Cl}$ 1,03 $-13,15, p=0,045)$

Conclusion: Thus, PD-synovitis has a prognostic value for increasing destructive radiographic changes.

References: no

Disclosure of Interests: None declared

DOI: 10.1136/annrheumdis-2020-eular.5200

$\mathrm{AB1076}$

COLOR DOPPLER HIGH- FREQUENCY ULTRASOUND OF DIGITAL ARTERIES IN PATIENTS WITH SYSTEMIC SCLEROSIS

O. Alekseeva ${ }^{1}$, N. Yudkina ${ }^{1}$, A. Demina ${ }^{1}$, A. Volkov ${ }^{1}$, E. Nasonov ${ }^{2} .{ }^{1}$ V.A. Nasonova Research Institute of Rheumatology, Diagnostic department, Moscow, Russian Federation; ${ }^{2}$ V.A. Nasonova Research Institute of Rheumatology, Moscow, Russian Federation

Background: Systemic sclerosis (SSc) can lead to vascular complications such as digital ulcers or pitting scars (DU/PS). These changes develop in most patients with SSc and exacerbate their condition. However, there are no methods for dynamic assessment of the vascular involvement. The dynamics of capillaroscopic changes is very slow.

Objectives: The aim of the study was to compare blood flow parameters of digital arteries in SSc patients and healthy individuals and to compare with nailfold capillaroscopy and clinical signs of ischemia (DU/PS).

Methods: 32 SSc patients, mean age 49,5 [42,0; 59,0] yrs and 26 'healthy', mean age 43,5 [33,0; 57,0], were included. Groups of patients differed by gender and age. The exclusion criterion was the presence of obliterating vascular disease of the upper extremities. An Esaote MyLab Twice US system with $22 \mathrm{MHz}$ linear probe was used. A total of SSc patients and controls underwent Color Doppler ultrasonography (CDUS) of $376(256+208)$ digital arteries to compare blood flow velocity, resistive indices (Rls) and presence of occlusion. Nailfold capillaroscopy, clinical and laboratory data were also evaluated.

Results: In digital arteries, pulsatility index (PI), peak systolic velocity (PSV) and end-diastolic velocity (EDV) were significantly lower and RI higher in SSc patients compared with controls (PSV: $13,28[9,88 ; 16,7]$ vs $17,45[12,65 ; 22,5]$ $\mathrm{cm} / \mathrm{s}, \mathrm{p}=0,008$; EDV: $2,68[1,78,4,05]$ vs $6,37[4,75 ; 8,5] \mathrm{cm} / \mathrm{s}, \mathrm{p}=0,000 ; \mathrm{Rl}: 0,78$ $[0,69 ; 0,81]$ vs $0,68[0,59 ; 0,74], p=0,005 ; \mathrm{PI}: 1,73[1,32 ; 2,19]$ vs $1,22[0,99$; $1,55], p=0,002)$.

We did not find any correlation between two methods. Also, we did not reveal any correlation between DU/PS, clinical, laboratory data and CDUS, but we found relationship between DU/PS and avascular areas or capillaroscopic findings ( $r=$ $0,37, p=0,045$ and $r=0,40, p=0,03$ correspondingly).

Conclusion: Blood flow is significantly decreased in digital arteries in SSc, but clinical features of vasculopathy depend on microcirculatory disorders. It is important to continue research to find methods for dynamic evaluation of microcirculatory changes.

References: no

Disclosure of Interests: None declared

DOI: 10.1136/annrheumdis-2020-eular.5395

\section{AB1077 $\quad$ VALIDATION OF A SIMPLIFIED SPANISH TOOL FOR SEMI-AUTOMATIC QUANTIFICATION OF SACROILIAC INFLAMMATION BY MAGNETIC RESONANCE IN SPONDYLOARTHRITIS (S- SCAISS).}

R. Almodovar ${ }^{1}$, P. Zarco-Montejo ${ }^{2}$, A. Bueno ${ }^{3}$, L. M. Molinero ${ }^{4}$ on behalf of SCAISS Study Group. ${ }^{1}$ Hospital Universitario Fundación Alcorcón, Madrid., Rheumatology Department., Madrid, Spain; ${ }^{2}$ Hospital Universitario Fundación Alcorcón, Madrid., Rheumatology Unit, Madrid, Spain; ${ }^{3}$ Hospital Universitario Fundación Alcorcón, Radiology Department, Madrid, Spain; ${ }^{4}$ ALCE ingeniería, Madrid, Spain

Background: With the objective to improve quantification of sacroiliitis maintaining a practical perspective, our group developed SCAISS, a semi-automated method to measure bone marrow edema (BME) in MR images from sacroiliac (SI) joints, combining semi-axial and semi-coronal slices [1]. The 2009 ASAS definition of active sacroiliitis was based on standard semi-coronal slices only, perpendicular semi-axial slices being considered but optional [2]. We hypothesized a simplified SCAISS (s- SCAISS) method using only semi-coronal slices.

Objectives: To analyze the validity and reliability and feasibility of a simplified Spanish tool for semi-automatic quantification of sacroiliac inflammation by magnetic resonance in spondyloarthritis (s- SCAISS) using a semi-coronal scan instead of combining semi-axial and semi-coronal slices.

Methods: The s- SCAISS was designed as an image-processing software. We performed the following analysis: (1) three readers evaluated SI images of 23 patients with axial SpA and various levels of BME severity with the s-SCAISS and SCAISS, and two non-automated methods, SPARCC and Berlin; (2) 20 readers evaluated 12 patients images, also with the three methods. Convergent validity, reliability and feasibility were estimated.

Results: The interobserver reliability (ICC and 95\% CI) in the three observers study was: $s-S C A I S S=0.69(0.490-0.845) ;$ SCAISS $=0.770(0.580-0.889)$; Berlin $=0.725(0.537-0.860)$; and SPARCC $=0.824(0.671-0.916)$. In the 20 observers' study, ICC was: $s-$ SCAISS $=0.66(0.478-0.863)$; SCAISS $=0.801$ (0.653-0.927); Berlin $=0.702(0.518-0.882)$; and SPARCC $=0.790(0.623$ 0.923). Spearman correlation coefficient between s- SCAISS_BERLIN was $r=$ 0.712 and s- SCAISS_SPARCC was $r=0.779$ and s- SCAISS_SCAISS was $r=$ 0.90. Similar results showed SCAISS BERLIN and SCAISS_SPARCC $(r=0.729$ and 0.840 ), respectively.

Conclusion: The simplified SCAISS (s-SCAISS) using only semi-coronal slice permits a valid, reliable, and fast calculation of overall BME lesion at the SI joint. References:

[1] Zarco P, Almodóvar R, Bueno Á, Molinero LM; SCAISS Study Group.Development and validation of SCAISS, a tool for semi-automated quantification of sacroilitis by magnetic resonance in spondyloarthritis. Rheumatol Int. 2018 Oct;38(10):1919-1926.

[2] Rudwaleit M, Jurik AG, Hermann KGA et al. Defining active sacroiliitis on magnetic resonance imaging (MRI) for classification of axial spondyloarthritis: a consensual approachby the ASAS/OMERACT MRI group. Ann Rheum Dis 2009; 68:1520-7.

Acknowledgments: Mireia Moreno (Rheumatology, H. Parc Taulí), Xavier Juanola (Rheumatology, H. Bellvitge), Maite Ventemillas (Radiology, H. Parc Taulí), Victoria Navarro (Rheumatology, H. La Paz), Daniel Bernabeu (Radiology, H. La Paz), Rafael Montero Perez-Barquero (Radiology, H. Reina Sofía de Córdoba), Concha Crespo (Radiology, $\mathrm{H}$. de San Juan de Alicante), Enrique Batlle (Rheumatology, H. de San Juan de Alicante), Carmen Castro Copete (Radiology, H. H. de San Juan de Alicante), Carlos Quiles (Radiology, $H$.

General Universitario de Valencia), Emma Beltrán (Rheumatology, H. del Mar) Fran García Lorente (Rheumatology, H. Universitario de Basurto), Fernando Díez (Radiology, H. Universitario de Basurto), Luis Linares (Rheumatology, H. Virgen de la Arrixaca), Manuel José Moreno Ramos (Rheumatology, H. Virgen de la Arrixaca), Angela Cepero (Radiology, H. H. Virgen de la Arrixaca), Cristina Fernández Carballido (Rheumatology, H. de Elda), Christopher Pack (Radiology, H. de Elda). Disclosure of Interests: RAQUEL ALMODOVAR Speakers bureau: Abbvie, Celgene, Janssen, Lilly, Novartis, Pfizer.

Pedro Zarco-Montejo Speakers bureau: Abbvie, MSD, Novartis, Pfizer., Angel Bueno: None declared, Luis Miguel Molinero: None declared DOI: 10.1136/annrheumdis-2020-eular.2134

\section{$\mathrm{AB} 1078$ USE OF MYOSITIS SPECIFIC AUTOANTIBODIES TEST ACROSS A LARGE NHS HOSPITAL TRUST}

J. Ball ${ }^{1}$, A. Radunovic ${ }^{2}$, C. Scott ${ }^{1}$, M. Stevens ${ }^{1} .{ }^{1}$ Barts Health NHS Trust, Immunology, London, United Kingdom; ${ }^{2}$ Barts Health NHS Trust, Neurology, London, United Kingdom

Background: The immunology laboratory at Barts Health supports a large clinical myopathy service, providing blood tests for myositis-specific autoantibodies (MSA) by a commercial line immunoblot panel for Jo1, PL7, PL12, SRP, Mi2, Ku $\mathrm{PM}-\mathrm{Scl}$ and $\mathrm{Scl}-70$.

As Idiopathic Inflammatory Myositis (IIM) disease subtype definitions have evolved from the 1975 Bohan \& Peter criteria, the discovery of new antibodies has proven useful in the hands of neuromuscular clinicians whose patients have a high pre-test probability of disease. Ready availability of the test has led to increased demand from:

1. Respiratory physicians with patients with severe Interstitial Lung Disease (ILD) which can be a symptom of some IIMs.

2. The connective tissue disease (CTD) screening section of the laboratory in which many patients are screened for antinuclear antibodies (ANA), which occasionally produces a pattern that may be associated with an MSA.

\section{Objectives:}

1. Determine the frequency of MSA requests from different departments.

2. To investigate the possibility of rejecting requests for MSAs at the laboratory in the absence of an elevated creatine kinase (CK), a hallmark of muscle damage associated with myositis (1)

Methods: MSA were measured by a commercial line blot (Bluediver) which included Jo-1, PL-7, PL-12, Mi-2, Ku, SRP-54 and PM-Scl-100. Demographics and results for all MSA requested between September 2017 and November 2019 were pulled from laboratory records, together with CK results (if performed). CK was interpreted as low, normal or elevated according to reference ranges of 25-200 U/L (female) or 40-320 U/L (male) 